\title{
Gravity wave reflection and its influence on the consistency of temperature- and wind-based momentum fluxes simulated above Typhoon Ewiniar
}

\author{
Y.-H. Kim ${ }^{1}$, H.-Y. Chun ${ }^{1}$, P. Preusse ${ }^{2}$, M. Ern ${ }^{2}$, and S.-Y. Kim ${ }^{3}$ \\ ${ }^{1}$ Department of Atmospheric Sciences, Yonsei University, Seoul, South Korea \\ ${ }^{2}$ Institute for Energy and Climate Research - Stratosphere (IEK-7), \\ Forschungszentrum Jülich, Jülich, Germany \\ ${ }^{3}$ Korea Institute of Atmospheric Prediction Systems, Seoul, South Korea \\ Correspondence to: H.-Y. Chun (chunhy@yonsei.ac.kr)
}

Received: 10 November 2011 - Published in Atmos. Chem. Phys. Discuss.: 29 February 2012

Revised: 7 October 2012 - Accepted: 9 November 2012 - Published: 16 November 2012

\begin{abstract}
For a case study of Typhoon Ewiniar performed with a mesoscale model, we compare stratospheric gravity wave $(\mathrm{GW})$ momentum flux determined from temperature variances by applying GW polarization relations and by assuming upward propagating waves, with GW momentum flux calculated from model winds which is considered as a reference. The temperature-based momentum-flux profile exhibits positive biases relative to the reference, which fluctuate significantly with altitude. The vertically-averaged magnitude of the positive biases is about $14 \%$ of the reference momentum flux. We found that this deviation from the reference stems from the interference between upward and downward propagating waves. The downward propagating GWs are due mainly to partial reflections of upward propagating waves at altitudes where the background wind and stability change with height. When the upward and downward propagating waves are decomposed and their momentum fluxes are calculated separately from temperature perturbations, the fraction of the momentum flux arising from the downward propagating waves is about $4.5-8.2 \%$ of that from the upward propagating waves. The net momentum flux of upward and downward propagating GWs agrees well with the reference from the model wind perturbations. The implications of this study for the GW momentum-flux observations from satellites are discussed.
\end{abstract}

\section{Introduction}

Gravity waves impact upon the general circulation of the middle atmosphere by deposition of their momentum and energy into the large-scale flow (Lindzen, 1981; Matsuno, 1982; Garcia and Solomon, 1985). The vertical flux of the momentum arising from gravity waves has been estimated by various measurement methods such as radiosonde, superpressure balloon, research aircraft, and radar. More recently, the global distribution of the gravity-wave momentum flux has been inferred from satellite observations of the temperature variance. Ern et al. (2004) first estimated the momentum flux from the satellite-observed temperature variance based on the linear theory of internal gravity waves, along with a simultaneous estimation of the vertical and horizontal wavelengths. They showed a good agreement between the global distribution of the absolute momentum flux obtained from the satellite and that from the gravity-wave parameterization by Warner and McIntyre (2001). The method of Ern et al. (2004) has been applied to various studies on gravity waves using satellite observations for both specific events and climatologies (e.g., Alexander et al., 2008; Wright et al., 2010; Ern et al., 2011).

Estimating GW momentum flux needs several processing steps which are sources of errors in the estimation (e.g., Zhang et al., 2004; Ern et al., 2004). The first step is the separation of GWs from the large-scale background state. This can be based either on a local approximation (e.g., polynomial fits to single vertical profiles; refer to Zhang et al., 
2004) or on the estimate of zonal mean and planetary-scale waves from the global observations (e.g., Ern et al., 2004, 2011). After separating GWs, the second step is estimating wave parameters. For current-day satellite observations only amplitudes and vertical wavelengths can be determined with a good accuracy, while the horizontal wavelength has to be estimated from phase differences between adjacent profiles. This is prone to aliasing, and the direction of the wave remains unknown. Accordingly, the error of GW momentumflux estimates from limb-sounding measurements is at least a factor of 2 (Ern et al., 2004). However, recent advances in detector technology make limb-imaging observations feasible, which would allow for three-dimensional (3-D) temperature measurements, if realized (Preusse et al., 2009). In this case, 3-D fitting techniques can be applied for the estimation of the momentum flux (Lehmann et al., 2012) and the currently dominating error sources vanish.

Error sources that have been neglected so far may become important in the GW momentum-flux estimation using 3-D temperature measurements such as future limb imagers: first, the assumption that all observed waves propagate upward, and second, the polarization relation between the wind and temperature perturbations employed to calculate the momentum flux. The assumption has been used for the momentumflux estimations of the observed gravity waves not only by the satellites but by other measurement methods as well (e.g., Espy et al., 2004; Hertzog et al., 2008; Li et al., 2011). However, in the middle atmosphere downward propagation of gravity waves occurs by reflection of the upward propagating waves due to the changes in the vertical wavenumber with height (e.g., Hines and Reddy, 1967; Gossard and Hooke, 1975) or from the in situ wave sources in the upper level (e.g., Holton and Alexander, 1999; Zhou et al., 2002; Chun and Kim, 2008). The downward propagating waves can modify the momentum flux significantly in both magnitude and spectral shape, as shown by Chun and Kim (2008). The polarization relation used for the momentum-flux estimations is based on the linear theory of mid-frequency gravity waves under the upward-propagation assumption.

In the present study, we examine the accuracy of the gravity-wave momentum-flux estimate using the temperature variance under the assumption that all the observed waves are propagating upward. For this, we use the three-dimensional mesoscale-model result of Typhoon Ewiniar (Kim et al., 2009) that includes both temperature and wind data. First, the gravity-wave momentum flux estimated from the temperature variance using the method of Ern et al. (2004) based on the upward-propagation assumption is compared to the momentum flux that is directly calculated from the horizontal and vertical wind perturbations. Then, the temperature perturbations are decomposed into upward propagating and downward propagating waves. The vertical profiles of the temperature-based momentum fluxes according to the upward and downward propagating components are calculated separately, and their sum is compared with the temperature- based momentum flux considering exclusively the upward propagating waves and the momentum flux directly calculated using wind perturbations. The origin of the downward waves is also discussed. Finally, the implications of the present results for satellite measurements of the gravity-wave momentum flux are discussed.

\section{Method}

\subsection{Data and momentum flux estimation}

The data used in this study are the results of a threedimensional numerical simulation of gravity waves generated by Typhoon Ewiniar (2006) from 01:00 UTC, 7 July to 06:00 UTC, 8 July. The simulation was conducted by Kim et al. (2009) using the Advanced Research WRF (Weather Research and Forecasting) model (Skamarock et al., 2005) with a horizontal grid spacing of $27 \mathrm{~km} .132$ vertical levels are used from the surface to $0.1 \mathrm{hPa}(z \sim 65 \mathrm{~km})$ with a vertical grid spacing of about $500 \mathrm{~m}$ in the stratosphere. The horizontal domains for the simulation and the wave analyses are illustrated in Fig. 1 of Kim and Chun (2010). The previous studies by Kim et al. (2009) and Kim and Chun (2010) showed that the stratospheric gravity waves above Typhoon Ewiniar in this simulation are in good agreement with those in European Centre for Medium-Range Weather Forecasts (ECMWF) analysis data and the Atmospheric Infrared Sounder (AIRS) observations in terms of the timing, location, propagation direction, and wavelength.

Gravity wave perturbations are obtained by subtracting the background states from the simulated variables. Here the background states are defined as $21 \times 21$ point $(567 \times 567 \mathrm{~km})$ horizontal running averages of the variables. The dependence of the results on the background states is discussed in Sect. 4. Hereafter, prime and overbar indicate the gravity-wave perturbation and the background state, respectively. Using the polarization relations between wind and temperature perturbations, the mean zonal-momentum flux $\left(F_{W}=\bar{\rho} \overline{u^{\prime} w^{\prime}}\right)$ of upward or downward propagating gravity waves can be expressed as

$F_{T}=-\frac{g^{2}}{2} \bar{\rho} \sum_{k, l, \omega} \frac{k}{m N^{2}} \frac{|\tilde{T}|^{2}}{\bar{T}^{2}}$,

where $u, w, T, \rho$, and $N$ are the zonal and vertical winds, temperature, density, and the background Brunt-Väisälä frequency, respectively. The tilde indicates a complex Fourier coefficient such that $T^{\prime}=\sum_{k, l, \omega} \operatorname{Re}\{\tilde{T} \exp [i(k x+l y-\omega t)]\}$, where $k, l$, and $\omega$ are the zonal and meridional wavenumbers and ground-based frequency, respectively. The vertical wavenumber $m$ is obtained from the dispersion relation of the mid-frequency gravity waves, $m^{2}=N^{2} / \hat{c}^{2}$, where $\hat{c}$ is the intrinsic phase speed. Note that the sign of $m$ is negative for 
upward propagating waves and positive for downward propagating waves, with the sign convention of non-negative $\omega$. For the calculation of $F_{T}$ in this study, we take the sign of $m$ to be negative, based on the assumption that the temperature variance stems from upward propagating waves, which is often made in other studies as well (e.g., Espy et al., 2004; Boccara et al., 2008; Hertzog et al., 2008; Li et al., 2011).

\subsection{Wave decomposition}

Internal gravity waves can be decomposed into upward and downward propagating components, and the Fourier coefficient of the temperature perturbation can be written as $\tilde{T}=\tilde{T}_{\text {up }}+\tilde{T}_{\text {down }}$, where the subscripts "up" and "down" indicate the upward and downward propagating components, respectively. For two-dimensional $(x-z)$ waves, the Fourier coefficients of horizontal winds can be obtained from the polarization relation of internal gravity waves as $\tilde{u}_{\text {up }}=A \tilde{T}_{\text {up }}$ and $\tilde{u}_{\text {down }}=-A \tilde{T}_{\text {down }}$, where $A=\operatorname{ig}(N \bar{T})^{-1}$ under the mid-frequency approximation and the WKB approximation. From the above equations, the Fourier coefficients of the upward and downward components of the temperature perturbation are obtained as

$\tilde{T}_{\text {up }}=(\tilde{T}+\tilde{u} A) / 2$,

$\tilde{T}_{\text {down }}=(\tilde{T}-\tilde{u} A) / 2$

where $\tilde{u}=A\left(\tilde{T}_{\text {up }}-\tilde{T}_{\text {down }}\right)$. This result is expanded to the three-dimensional waves by replacing $\tilde{u}$ in Eq. (2) by $(\tilde{u}, \tilde{v})$. $\boldsymbol{K} /|\boldsymbol{K}|$, where $\boldsymbol{K}$ is the horizontal wavenumber vector. Then, the momentum flux considering both the upward and downward propagating gravity waves can be obtained as

$F_{T_{\text {total }}}=F_{T_{\text {up }}}+F_{T_{\text {down }}}$

$=-\frac{g^{2}}{2} \bar{\rho} \sum_{k, l, \omega} \frac{k}{m N^{2}} \frac{\left|\tilde{T}_{\text {up }}\right|^{2}-\left|\tilde{T}_{\text {down }}\right|^{2}}{\bar{T}^{2}}$

where the sign of $m$ is the same as that used in Eq. (1), i.e., negative with the sign convention of non-negative $\omega$. The difference between Eqs. (1) and (3) is that Eq. (3) uses the difference of the temperature variances from upward and downward propagating waves rather than the total temperature variances.

\section{Results}

Figure 1a shows profiles of the zonal-momentum flux calculated directly from the wind perturbations $\left(F_{W}\right)$ and estimated from the temperature variances considering only the upward propagating gravity waves $\left(F_{T}\right)$. Both profiles represent a consistently decreasing trend of the momentum flux with altitude, which is due mainly to the radiative and turbulent dissipation of waves (for details, see Marks and Eckermann, 1995; Kim et al., 2005). The magnitude of $F_{T}$ is,
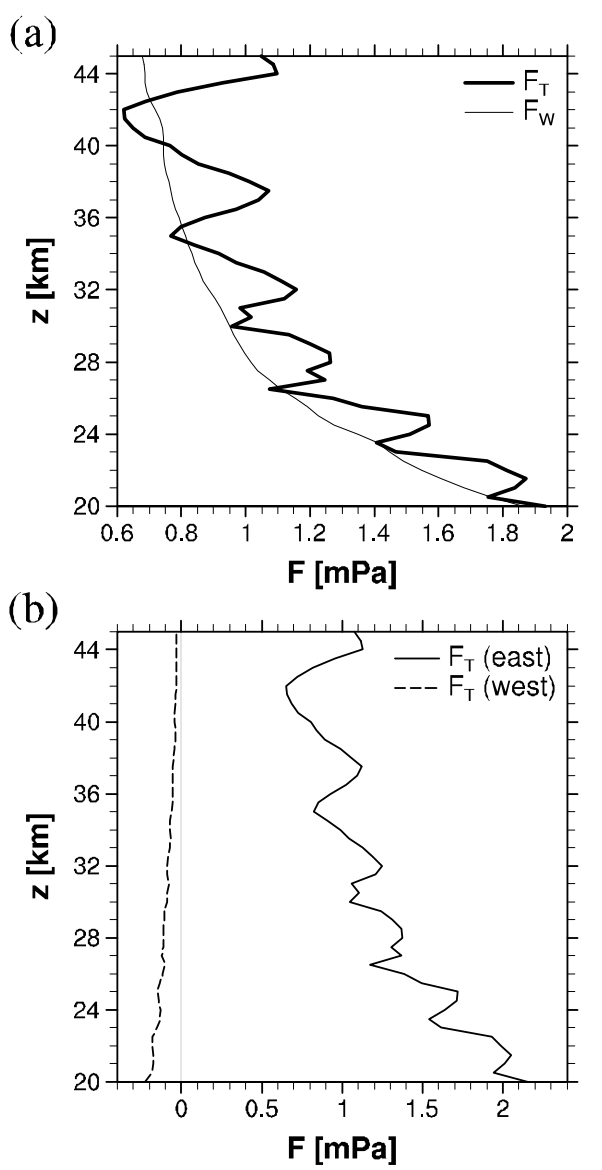

Fig. 1. Momentum-flux profiles (a) estimated from temperature variances (thick) and calculated from wind perturbations (thin) and (b) estimated from temperature variances for eastward (solid) and westward propagating (dashed) waves.

however, larger than that of $F_{W}$ at most altitudes, and it fluctuates vertically. The difference between $F_{T}$ and $F_{W}$ is in the range of $0.25-0.34 \mathrm{mPa}\left(17-39 \%\right.$ of $\left.F_{W}\right)$ at $z=22,25,28.5$, 32 , and $37.5 \mathrm{~km}$ and is $0.40 \mathrm{mPa}\left(60 \%\right.$ of $\left.F_{W}\right)$ at $z=44 \mathrm{~km}$, and the vertically averaged difference is $0.14 \mathrm{mPa}(14 \%$ of $\left.F_{W}\right)$. Figure $1 \mathrm{~b}$ shows profiles of $F_{T}$ for eastward $(\hat{c}>0)$ and westward propagating $(\hat{c}<0)$ waves. The momentum flux of the westward waves is much smaller than that of the eastward waves, because most westward waves are filtered out by the stratospheric easterly winds (see Fig. 5a) in the summertime. As the momentum flux of the eastward waves is dominant and fluctuates with amplitudes similar to $F_{T}$ shown in Fig. 1a, we hereafter present the results only for the eastward waves.

To clarify the origin of the vertical fluctuations in $F_{T}$, structures in the temperature variances are investigated. Figure 2a shows the $x-z$ cross section of temperature variances for the purely eastward propagating waves (i.e., $\varphi=0$, where $\varphi$ is the azimuthal angle of wave-propagation direction) at 00:00 UTC, 8 July. The variance signals are slanted eastward 

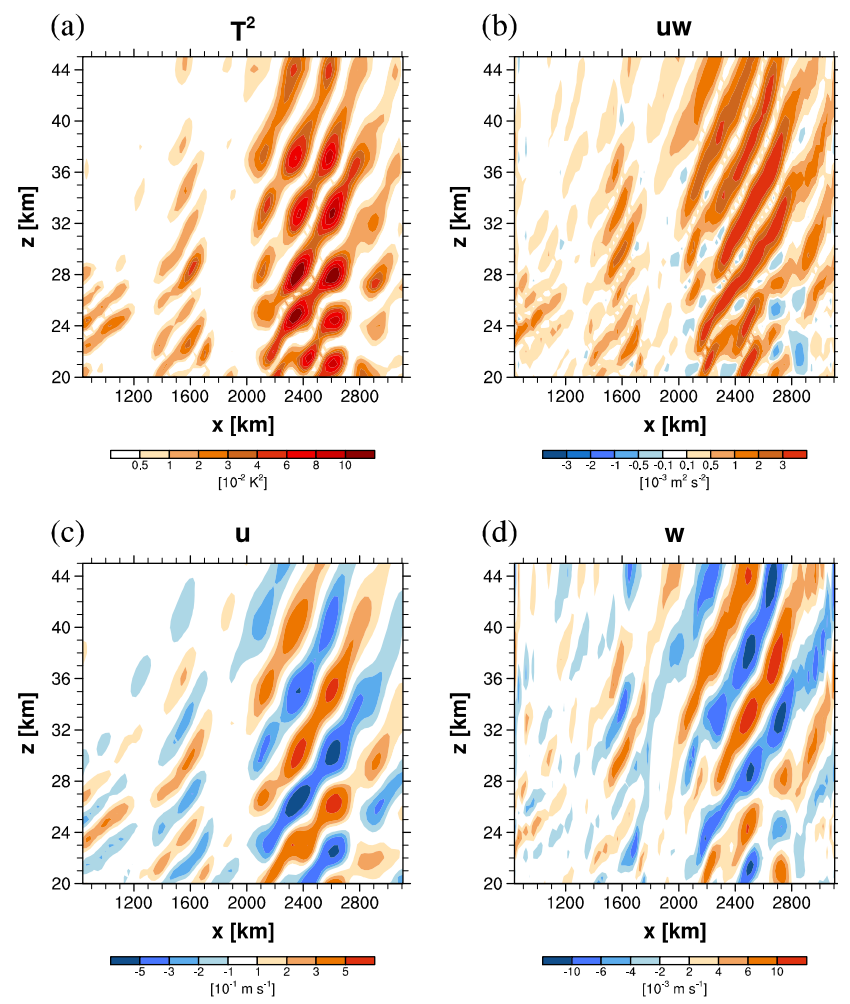

Fig. 2. $x-z$ cross sections of (a) temperature variances, (b) covariances between the zonal and vertical wind perturbations, (c) zonal wind perturbation, and (d) vertical wind perturbation for purely eastward propagating waves (waves propagating along the $\mathrm{x}$-axis) at 00:00 UTC, 8 July. The variables are normalized by the basic density in (a) and (b) and by the square root of the basic density in (c) and (d).

and reveal several nodes at fixed altitudes of $23,26.5,30.5$, 35 , and $42 \mathrm{~km}$ in $x>2000 \mathrm{~km}$. Note that the altitudes of the nodes correspond to the local minima of $F_{T}$ as shown in Fig. 1. These imply that waves slanted westward are superimposed with non-negligible amplitudes on the eastward slanted waves, and that the interference between the eastward and westward slanted waves is responsible for the vertical fluctuations in the profile of $F_{T}$. Given that only eastward propagating waves are considered here, a westward slant of waves indicates downward propagation.

Indeed, the vertical node structure also appears in the variances of other single variables, whereas it does not appear in the covariance between $u^{\prime}$ and $w^{\prime}$. Between the variance and the covariance, two different features are found in their two-dimensional fields (Fig. 2a and b): first, the node structure is less prominent in the covariance field, and second, there are negative values of covariance between the regions of large positive covariance. The former can be explained by locations of local maxima and nodes in the fields of $u^{\prime}$ and $w^{\prime}$ (Fig. 2c and d). For the eastward slanted waves, the two variables are in phase, which result in the positive covari-

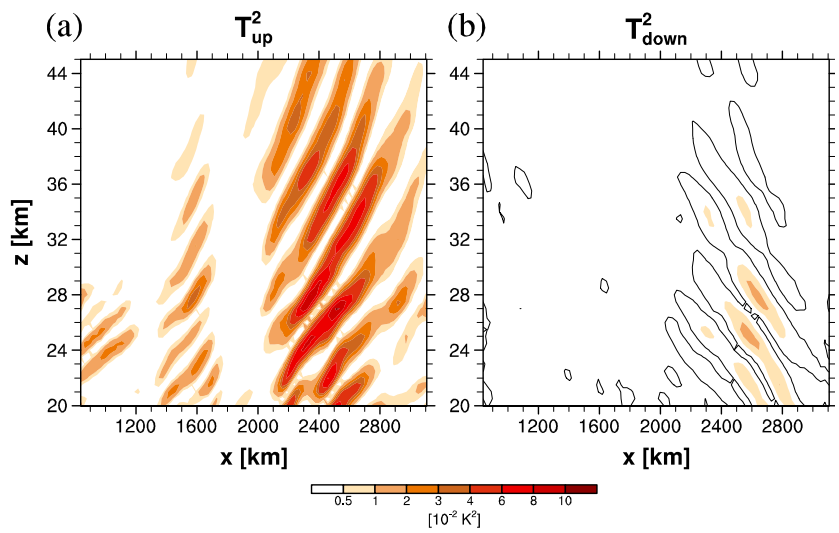

Fig. 3. The same as in Fig. 2a except for variances from decomposed components of (a) upward and (b) downward waves. In (c), regions of $10^{-3} \mathrm{~K}^{2}$ are plotted with black contours.

ance. Along the phase lines, there exist nodes in $u^{\prime}$ where local maxima of $w^{\prime}$ are located, and vice versa, resulting that the covariance between $u^{\prime}$ and $w^{\prime}$ has less prominent node structure. Those two features in the covariance field (Fig. 2b) cause the differences between the zonal-mean variance and covariance profiles (and thus, $F_{T}$ and $F_{W}$ ). In addition, zonal mean of the covariance between $u^{\prime}$ and $T^{\prime}$, which should be zero for either upward propagating or downward propagating gravity waves, is also calculated (not shown). In our case, this covariance is non-zero and it fluctuates vertically, indicating the superposition of the upward propagating and downward propagating waves (Dunkerton, 1995). Note that the negative momentum flux for the eastward propagating waves in Fig. $2 b$ also indicates the existence of downward propagating waves.

To investigate the downward propagating waves quantitatively, temperature fields are decomposed into upward and downward waves, as shown in Eq. (2), and the $x-z$ cross sections of variances in the decomposed temperature fields are presented in Fig. 3a and b for the zonal waves at the same time as in Fig. 2a. As expected, the downward waves coexist with the upward waves in $x>2000 \mathrm{~km}$. Both waves have similar dominant horizontal wavelengths of about $500 \mathrm{~km}$, but various vertical wavelengths with height ranging from 6 to $15 \mathrm{~km}$. The temperature variance of the downward waves is about $7.9 \%$ of that of the upward waves. It is noteworthy that even though the variance of the downward waves is much smaller than that of the upward waves, effects of the interference between the two waves on the total variance fields can be significant, as shown in Fig. 2a. The $x-z$ cross sections at other times (not shown) indicate that the ratio of the mean variance of the downward waves to upward waves is about $8.2 \%$, and that the altitudes of nodes are not time-dependent during 06:00 UTC, 7 July-00:00 UTC, 8 July.

The eastward propagating waves illustrated in Fig. 3 are the most abundant component for both the upward and 
downward waves in this simulation. For waves propagating in other directions $(\varphi \neq 0$, not shown), the horizontal wavelength analysis shows broad spectra (200-660 km) with much smaller amplitudes than the zonal waves. The altitudes of nodes for these waves are relatively irregular and different from those for the zonal waves, which might result from the broad spectra of waves. Regardless of the different characteristics, the ratio of variances of the downward waves to the upward waves is about $4-8 \%$, which is consistent with that by the eastward propagating waves.

To quantify the difference in the momentum flux from Eqs. (1) and (3), Fig. 4 shows the profiles of the momentum fluxes for the upward and downward propagating waves as well as the sum of the two. The momentum flux of the downward propagating waves is $0.1 \mathrm{mPa}$ at $z=20 \mathrm{~km}$. This decreases at higher altitudes and oscillates with amplitudes of $\sim 0.005 \mathrm{mPa}$ (Fig. $4 \mathrm{~b}$ ). The ratio of the momentum fluxes of the downward waves to upward waves is $4.5-8.2 \%$. The net momentum flux $\left(F_{T_{\text {total }}}\right)$ is quite close to $F_{W}$. This confirms that the errors introduced in Fig. 1 are due mostly to ignoring the interference between the upward and downward waves in the variance fields.

The good agreement between $F_{T_{\text {total }}}$ and $F_{W}$ also confirms that the application of polarization relations inferred from the linear gravity wave theory does not cause notable errors. The polarization relations are derived with two approximations, i.e., WKB and mid-frequency. As justified by Wright et al. (2010), the mid-frequency approximation is valid for gravity waves with horizontal and vertical wavelengths of $\sim 100$ and $\sim 10 \mathrm{~km}$, respectively. The gravity waves considered in the present study are within those wavelengths, and we found that a relaxation of the mid-frequency approximation does not change the results (not shown). In order to examine the validity of the WKB approximation, we checked whether the present gravity waves satisfy the criterion of $\delta \equiv\left|m_{z z} / 2 m^{3}-3 m_{z} / 4 m^{4}\right| \ll 1$ proposed by Einaudi and Hines (1970). For a dominant wave in our case (a zonal wave with a phase speed of $10 \mathrm{~m} \mathrm{~s}^{-1}$ ), the maximum value of $\delta$ is about 0.26 , which is comfortably less than unity.

\section{Discussion}

The downward propagating waves identified in this case have amplitudes of $21-29 \%$ of the upward propagating waves, based on the magnitude of their momentum flux compared with that of the upward propagating waves (4.5-8.2\%). As no apparent wave sources in the upper stratosphere are found and the characteristics of the downward propagating waves are similar to those of the upward waves, the downward propagation might be related to the reflection of the upward propagating gravity waves generated below. Figure 5 shows the background zonal winds $(U)$, Brunt-Väisälä frequencies $(N)$, and the corresponding squared vertical wavenumbers $\left(\mathrm{m}^{2}\right)$ obtained from the dispersion relation of a wave prop- (a)

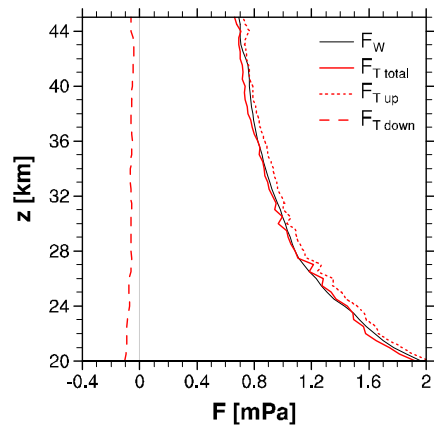

(b)

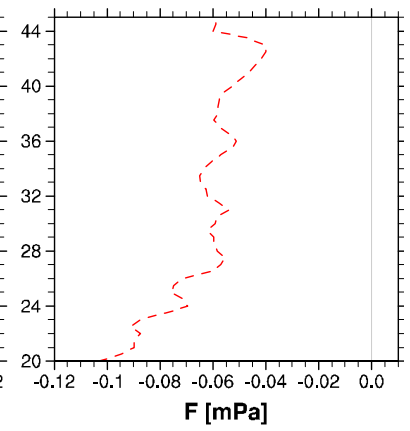

Fig. 4. (a) Momentum-flux profiles estimated from temperature variances by upward (red, dotted) and downward waves (red, dashed) for eastward propagating waves, and the sum of the two (red, solid). The momentum-flux profile calculated from wind perturbations for eastward propagating waves is also plotted (black, solid). The momentum-flux profile from temperature variances by downward waves is plotted again with an enlarged scale in (b).

agating eastward at a phase speed of $10 \mathrm{~m} \mathrm{~s}^{-1}$, which is the most dominant wave revealed in Figs. 2 and 3. As the background zonal winds have negative shear in the stratosphere, $m^{2}$ of the eastward propagating wave has a decreasing trend with altitude $\left(10 \times 10^{-7}-2 \times 10^{-7} \mathrm{rad}^{2} \mathrm{~m}^{-2}\right)$. Note that for any waves in the mid-frequency range, $m^{2}$ is larger than zero, indicating that total reflection of waves does not take place. However, there are significant changes in $m^{2}$ at several altitudes where $N$ sharply decreases, and it implies the existence of partial reflection of waves. For example, at $z=27-30.5 \mathrm{~km}, \mathrm{~m}^{2}$ changes from $8.6 \times 10^{-7} \mathrm{rad}^{2} \mathrm{~m}^{-2}$ to $4.0 \times 10^{-7} \mathrm{rad}^{2} \mathrm{~m}^{-2}$. If no reflection above $z=30.5 \mathrm{~km}$ is assumed, a simple two-layer model reveals the reflection coefficient as $|\Delta \mathrm{m} / 2 \bar{m}| \approx 0.19$ below $z=27 \mathrm{~km}$, where the numerator and denominator indicate the difference and sum of the vertical wavenumbers in the two layers, respectively (Eliassen and Palm, 1960). Here, the reflection coefficient is the amplitude ratio of the downward waves to the upward waves.

To examine the contribution of the variations in $N$ to the wave reflection more quantitatively, the Taylor-Goldstein equation is solved for the dominant gravity wave obtained in the present simulation with a horizontal wavelength of $500 \mathrm{~km}$ and a phase speed of $10 \mathrm{~m} \mathrm{~s}^{-1}$,

$\frac{\partial^{2} \hat{w}}{\partial z^{2}}+\left[\frac{N^{2}}{\hat{c}^{2}}+\frac{1}{\hat{c}} \frac{\mathrm{d}^{2} U}{\mathrm{~d} z^{2}}-k^{2}\right] \hat{w}=0$,

for two different profiles of $N$, one as in Fig. 5a and the other smoothed linearly. Note that the profile of $U$ is used as in Fig. 5a for both calculations. Here, $\hat{w}$ indicates a complex Fourier coefficient of vertical wind normalized by the square root of the basic density. To specify the upper boundary conditions of $\hat{w}$ and $\partial \hat{w} / \partial z$, the WKB solution of Eq. (4) and its 
(a)

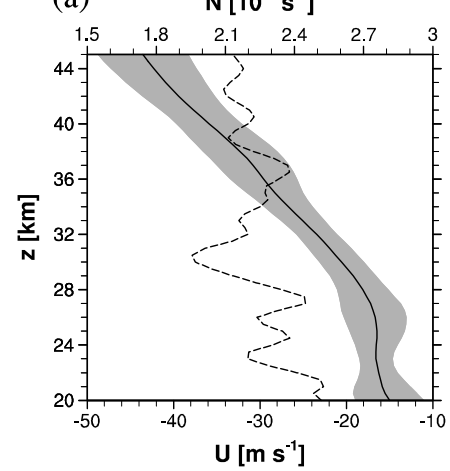

(b)

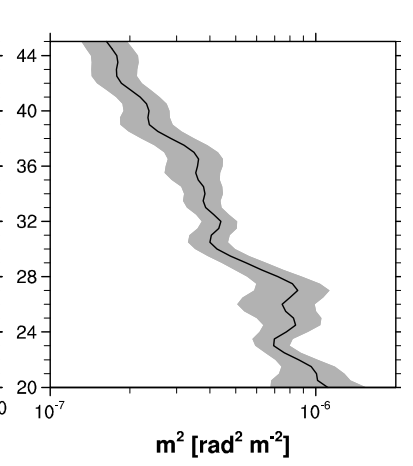

Fig. 5. Profiles of (a) the horizontal and temporal mean zonal wind (solid) and Brunt-Väisälä frequency (dashed) and (b) corresponding squared vertical wavenumber for purely eastward propagating waves at a phase speed of $10 \mathrm{~m} \mathrm{~s}^{-1}$ (solid). Shading indicates ranges within one standard deviation of the background zonal winds in (a) and of the squared vertical wavenumbers in (b) from their means.

vertical derivative,

$\hat{w}=C\left[\exp \left(-i|m| z_{\mathrm{r}}\right)+R \exp \left(i|m| z_{\mathrm{r}}\right)\right]$,

$\frac{\partial \hat{w}}{\partial z}=-C i|m|\left[\exp \left(-i|m| z_{\mathrm{r}}\right)-R \exp \left(i|m| z_{\mathrm{r}}\right)\right]$

are applied, where $z_{\mathrm{r}}=z-z_{0}, z_{0}$ is a reference altitude at which the vertical variation of the background state is not rapid, and $|m|$ is the square root of the term in the bracket in Eq. (4). $C$ and $R$ are complex values of which the absolute values are the amplitude of the upward-propagating waves and the reflection coefficient, respectively. Note that Eq. (5) is valid only near the reference altitude $z_{0}$ (i.e., $z_{\mathrm{r}} \sim 0$ ), and far from this altitude, Eq. (4) should be solved numerically. By letting $z_{0}=45 \mathrm{~km}$, the boundary conditions of Eq. (4) at $45 \mathrm{~km}$ can be specified by $\hat{w}=C\left(1+R_{\mathrm{t}}\right)$ and $\partial \hat{w} / \partial z=-C i\left|m_{\mathrm{t}}\right|\left(1-R_{\mathrm{t}}\right)$, where the subscript " $\mathrm{t}$ " indicates values at $45 \mathrm{~km}$. After specifying that $R_{\mathrm{t}}=0$ (i.e., no reflected waves are assumed at $45 \mathrm{~km}$ ), Eq. (4) is integrated from $z=45$ to $20 \mathrm{~km}$ using Bulirsch-Stoer method (Press et al., 1992). Figure 6 shows the vertical wind fields obtained for the two profiles of $N$ without and with smoothing. Note that the fields are normalized by the square root of the basic density and by the amplitude of the upward propagating waves at $20 \mathrm{~km}$. Being consistent with the simulation result, the vertical node structure appears in the vertical wind field for the non-smoothed $N$ case, whereas it does not for the smoothed $N$ case. The reflection coefficient at $20 \mathrm{~km}$ is calculated using $\hat{w}$ and $\partial \hat{w} / \partial z$ obtained from Eq. (4), by applying Eq. (5) with $z_{0}=20 \mathrm{~km}$. The reflection coefficients at $20 \mathrm{~km}$ are 0.18 and 0.01 for the non-smoothed and smoothed cases, respectively. This result confirms that the partial reflection by the vertical variation in $N$ explains a large portion of the downward propagating waves in our simulation. (a)

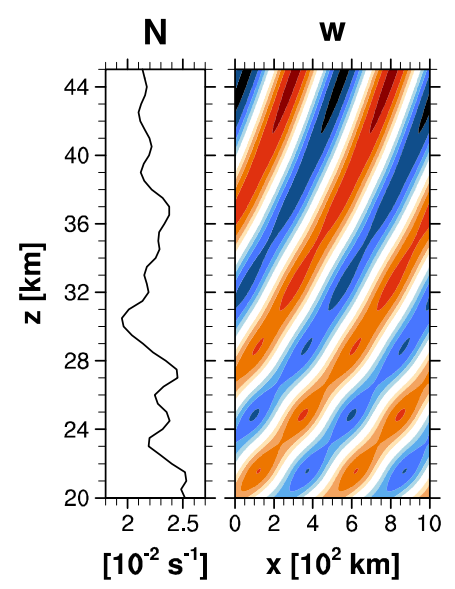

(b)

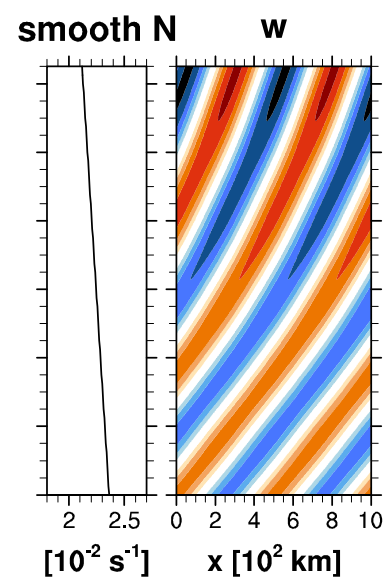

Fig. 6. $x-z$ cross sections of the vertical wind perturbations obtained from the Taylor-Goldstein equation for the dominant eastward propagating wave with the horizontal wavelength of $500 \mathrm{~km}$ at a phase speed of $10 \mathrm{~m} \mathrm{~s}^{-1}$ for the profiles of the Brunt-Väisälä frequency (a) without and (b) with smoothing. The perturbations are normalized by the amplitude of the upward propagating waves at $20 \mathrm{~km}$ and by the square root of the basic density, and the interval in shading is 0.3 . The profiles of the Brunt-Väisälä frequency used are also plotted in the left of each cross section.

The downward propagating gravity waves in the stratosphere have been detected from in situ measurements (e.g., Yoshiki and Sato, 2000; Yamamori and Sato, 2006; Sato and Yoshiki, 2008) utilizing analysis methods such as hodograph or two-dimensional vertical wavenumber-frequency spectrum. Using radiosonde measurements, Yoshiki and Sato (2000) and Sato and Yoshiki (2008) observed the downward propagating gravity waves around the stratospheric polar night jet that are generated from the upper-stratosphere jet by a spontaneous adjustment process. Recently, Sato et al. (2012) proposed the partial reflection mechanism by a rapid change in the static stability for the downward propagating waves near the stratospheric polar jet simulated in a high-resolution general circulation model. It is noteworthy, however, that in the conventional sounding analysis, it is non-trivial to extract the background state from the observed profile (e.g., Zhang et al., 2004), especially the background state that changes rapidly with height. This makes it difficult for the partial reflection mechanism to be confirmed from the observed gravity waves compared with the simulated gravity waves.

There is also a possibility of artificial wave reflection at the upper boundary of the numerical model. By using the damping layer ( $z=45-65 \mathrm{~km}$ in the present case) with a depth greater than the vertical wavelength of the waves, the artificial reflection near the upper boundary can be minimized. Based on the calculations by Klemp and Lilly (1978), the expected coefficient of the artificial reflection by the damping 
layer is only about 0.05 or less in our case. The actual coefficient of reflection at $z=45 \mathrm{~km}$, however, appears to be much larger $(\sim 0.28)$ than that value. There are two possibilities of obtaining the larger coefficient at the physical model top $(z=45 \mathrm{~km})$ : (i) artificial reflection at $z=45 \mathrm{~km}$ in the present simulation can be larger than that predicted in the analytical study by Klemp and Lilly (1978) based on a simple dynamic model, and (ii) the internal partial reflections might also occur above $z=45 \mathrm{~km}$, especially near the stratopause. The relative importance of the two processes for the amount of downward propagating waves at $z=45 \mathrm{~km}$ is not clear at the moment.

In this study, the vertical-mean magnitude of the bias is not so large $(\sim 14 \%)$. However, the mean bias becomes much more significant when greater portions of the wave spectrum are partially reflected or some dominant waves experience total reflections, from which $m^{2}$ becomes zero under a favorable setting of the basic-state wind and stability. This may, for instance, happen in strong vertical wind gradients associated with the winter polar vortex. Total reflection of short horizontal-scale waves is likely to occur below the maximum of the polar night jet (e.g., Preusse et al., 2008), while partial reflection may occur both below and above the maximumwind altitude.

In Sect. 2.1, the gravity wave perturbations are obtained by subtracting the background states that are defined as the horizontal running averages. In general, the characteristics and magnitudes of the gravity wave perturbations can be sensitive to the background states. In order to examine the dependence of the present results on the background states, we repeat the whole analyses using the background states that are obtained from the two-dimensional $(x-y)$ 2nd-order polynomial fits. Note that for this case, the non-gravity wave components $(|\hat{\omega}| \leq|f|$ or $|\hat{\omega}| \geq N$, where $\hat{\omega}$ and $f$ are the intrinsic frequency and the Coriolis parameter, respectively) are additionally filtered from the perturbations. The results from the two methods are generally similar, although the magnitudes of the momentum fluxes for the polynomial-fit method are $\sim 10 \%$ larger (not shown), and the main conclusion of the present study is not dependent on the background states.

\section{Conclusions}

Based on mesoscale modeling results of Typhoon Ewiniar (Kim et al., 2009), momentum flux values inferred from temperature variances by means of the GW polarization relations are compared to a reference GW momentum flux that calculated from the model winds. The momentum flux estimated from the temperatures exhibits vertical fluctuations and positive biases with peaks of $17-39 \%$ at $z=20-40 \mathrm{~km}$ and $60 \%$ at $z \sim 45 \mathrm{~km}$. The deviations stem from the interference between the upward and downward propagating waves, which is ignored in the original estimation by assuming all waves to propagate upward. The upward and downward waves are decomposed using the polarization relation between temperature and horizontal-wind perturbations, and the momentum flux for each wave is re-estimated. The relative magnitude of the momentum flux of the downward waves to upward waves is $4.5-8.2 \%$. The downward propagation of waves can be explained to a large extent by the partial reflection of the upward waves at various altitudes due to the changes in the vertical wavenumber with height, mainly by the static stability, along with a minor contribution by the artificial reflection from a model dissipation layer.

In the present study, we performed wave analyses in the horizontal direction using the Fourier transform rather than in the vertical direction, allowing us to estimate the momentum flux of each altitude independently. If the data were analyzed by a method using vertical analysis windows, the vertical fluctuations in the temperature-based momentum flux might be much smoothed. However, the vertical-mean momentum flux would still have the positive biases with a magnitude similar to that in the present $(\sim 14 \%)$, assuming that the vertical and horizontal wavelengths estimated using the vertical analysis windows are also similar to those in the present analysis. Note that this assumption can be satisfied only when the horizontal sampling number is large enough to estimate the horizontal wavelength accurately (Ern et al., 2004). In principle, this positive bias remains, unless (i) the eastward and westward slanted waves are separated by simultaneous wave analysis in horizontal and vertical directions, (ii) the upward and downward waves are decomposed, for example by the method used here, or (iii) the covariance of two variables (e.g., $u^{\prime}$ and $T^{\prime}$ ) is used to estimate the momentum flux. The last two approaches require simultaneous observations of wind and temperature profiles, which is not available from satellites at present. Also the first method cannot be applied to present-day limb sounders as the horizontal sampling is too sparse, but might be applicable for future limb imaging missions (Lehmann et al., 2012). In the case discussed in this study, the fraction of downward propagating waves due to partial reflection on sharp gradients of the buoyancy frequency is rather small. Larger effects may be expected below strong wind gradients associated with the polar winter jets.

The magnitude of errors arising from the assumption of upward propagation of GWs may be smaller than that from the other error sources in the estimation of GW momentum flux using current-day limb sounders. However, considering that the currently dominant errors would be largely reduced in future limb imagers, the present study could be important for the GW momentum-flux estimation.

Acknowledgements. Part of this work was funded by ESA under the grant "Observation of Gravity Waves from Space", contract number: 22561/09/NL/AF. YHK and HYC were supported by the Korean Meteorological Administration Research and Development Program under Grant CATER-2012-3054.

Edited by: T. J. Dunkerton 


\section{References}

Alexander, M. J., Gille, J., Cavanaugh, C., Coffey, M., Craig, C., Eden, T., Francis, G., Halvorson, C., Hannigan, J., Khosravi, R., Kinnison, D., Lee, H., Massie, S., Nardi, B., Barnett, J., Hepplewhite, C., Lambert, A., and Dean, V.: Global estimates of gravity wave momentum flux from High Resolution Dynamics Limb Sounder observations, J. Geophys. Res., 113, D15S18, doi:10.1029/2007JD008807, 2008.

Boccara, G., Hertzog, A., Vincent, R. A., and Vial, F.: Estimation of gravity wave momentum flux and phase speeds from quasiLagrangian stratospheric balloon flights Part I, J. Atmos. Sci., 65, 3042-3055, 2008.

Chun, H.-Y. and Kim, Y.-H.: Secondary waves generated by breaking of convective gravity waves in the mesosphere and their influence in the wave momentum flux, J. Geophys. Res., 113, D23107, doi:10.1029/2008JD009792, 2008.

Dunkerton, T. J.: Horizontal buoyancy flux of internal gravity waves in vertical shear, J. Meteorol. Soc. Jpn., 73, 747-755, 1995.

Einaudi, F. and Hines, C. O.: WKB approximation in application to acoustic-gravity waves, Can. J. Phys., 48, 1458-1471, 1970.

Eliassen, A. and Palm, E.: On the transfer of energy in stationary mountain waves, Geofys. Publ.., 22, 23 pp., 1960.

Ern, M., Preusse, P., Alexander, M. J., and Warner, C. D.: Absolute values of gravity wave momentum flux derived from satellite data, J. Geophys. Res., 109, D20103, doi:10.1029/2004JD004752, 2004.

Ern, M., Preusse, P., Gille, J. C., Hepplewhite, C. L., Mlynczak, M. G., Russell III, J. M., and Riese, M.: Implications for atmospheric dynamics derived from global observations of gravity wave momentum flux in stratosphere and mesosphere, J. Geophys. Res., 116, D19107, doi:10.1029/2011JD015821, 2011.

Espy, P. J., Jones, G. O. L., Swenson, G. R., Tang, J., and Taylor, M. J.: Seasonal variations of the gravity wave momentum flux in the Antarctic mesosphere and lower thermosphere, J. Geophys. Res., 109, D23109, doi:10.1029/2003JD004446, 2004.

Garcia, R. R. and Solomon, S.: The effect of breaking gravity waves on the dynamics and chemical composition of the mesosphere and lower thermosphere, J. Geophys. Res., 90, 3850-3868, 1985.

Gossard, E. E. and Hooke, W. H.: Waves in the Atmosphere, Elsevier, Amsterdam, 456 pp., 1975.

Hertzog, A., Boccara, G., Vincent, R. A., Vial, F., and Cocquerez, P.: Estimation of gravity wave momentum flux and phase speeds from quasi-Lagrangian stratospheric balloon flights Part II, J. Atmos. Sci., 65, 3056-3070, 2008.

Hines, C. O. and Reddy, C. A.: On the propagation of atmospheric gravity waves through regions of wind shear, J. Geophys. Res., 72, 1015-1034, 1967.

Holton, J. R. and Alexander, M. J.: Gravity waves in the mesosphere generated by tropospheric convection, Tellus, 51, 45-58, 1999.

Kim, S.-Y. and Chun, H.-Y.: Momentum flux of stratospheric gravity waves generated by Typhoon Ewiniar (2006), Asia-Pac, J. Atmos. Sci., 46, 199-208, 2010.

Kim, S.-Y., Chun, H.-Y., and Baik, J.-J.: A numerical study of gravity waves induced by convection associated with Typhoon Rusa, Geophys. Res. Lett., 32, L24816, doi:10.1029/2005GL024662, 2005.

Kim, S.-Y., Chun, H.-Y., and Wu, D. L.: A study on stratospheric gravity waves generated by Typhoon Ewiniar: Numerical simulations and satellite observations, J. Geophys. Res., 114, D22104,
doi:10.1029/2009JD011971, 2009.

Klemp, J. B. and Lilly, D. K.: Numerical simulation of hydrostatic mountain waves, J. Atmos. Sci., 35, 78-107, 1978.

Lehmann, C. I., Kim, Y.-H., Preusse, P., Chun, H.-Y., Ern, M., and Kim, S.-Y.: Consistency between Fourier transform and smallvolume few-wave decomposition for spectral and spatial variability of gravity waves above a typhoon, Atmos. Meas. Tech., 5, 1637-1651, doi:10.5194/amt-5-1637-2012, 2012.

Li, Z., Liu, A. Z., Lu, X., Swenson, G. R., and Franke, S. J.: Gravity wave characteristics from $\mathrm{OH}$ airglow imager over Maui, J. Geophys. Res., 116, D22115, doi:10.1029/2011JD015870, 2011.

Lindzen, R. S.: Turbulence and stress owing to gravity wave and tidal breakdown, J. Geophys. Res., 86, 9709-9714, 1981.

Marks, C. J. and Eckermann, S. D.: A three-dimensional nonhydrostatic ray-tracing model for gravity waves: Formulation and preliminary results for the middle atmosphere, J. Atmos. Sci., 52, 1959-1984, 1995.

Matsuno, T.: A quasi one-dimensional model of the middle atmosphere circulation interacting with internal gravity waves, J. Meteorol. Soc. Jpn., 60, 215-226, 1982.

Press, W. H., Flannery, B. P., Teukolsky, S. A., and Vetterling, W. T.: Richardson extrapolation and the Bulirsch-Stoer method, in: Numerical Recipes in FORTRAN: The Art of Scientific Computing, Cambridge University Press, Cambridge, England, 718-725, 1992.

Preusse, P., Eckermann, S. D., and Ern, M.: Transparency of the atmosphere to short horizontal wavelength gravity waves, J. Geophys. Res., 113, D24104, doi:10.1029/2007JD009682, 2008.

Preusse, P., Schroeder, S., Hoffmann, L., Ern, M., Friedl-Vallon, F., Ungermann, J., Oelhaf, H., Fischer, H., and Riese, M.: New perspectives on gravity wave remote sensing by spaceborne infrared limb imaging, Atmos. Meas. Tech., 2, 299-311, doi:10.5194/amt-2-299-2009, 2009.

Sato, K. and Yoshiki, M.: Gravity wave generation around the polar vortex in the stratosphere revealed by 3-hourly radiosonde observations at Syowa station, J. Atmos. Sci., 65, 3719-3735, 2008.

Sato, K., Tateno, S., Watanabe, S., and Kawatani, Y.: Gravity wave characteristics in the Southern Hemisphere revealed by a highresolution middle-atmosphere general circulation model, J. Atmos. Sci., 69, 1378-1396, 2012.

Skamarock, W. C., Klemp, J. B., Dudhia, J., Gill, D. O., Barker, D. M., Wang, W., and Power, J. G.: A description of the Advanced Research WRF Version 2, Tech. Note NCAR/TN-486+STR, Boulder, CO, Natl. Cent. for Atmos. Res., 88 pp., 2005.

Warner, C. D. and McIntyre, M. E.: An ultrasimple spectral parameterization for nonorographic gravity waves, J. Atmos. Sci., 58, 1837-1857, 2001.

Wright, C. J., Osprey, S. M., Barnett, J. J., Gray, L. J., and Gille, J. C.: High Resolution Dynamics Limb Sounder measurements of gravity wave activity in the 2006 Arctic stratosphere, J. Geophys. Res., 115, D02105, doi:10.1029/2009JD011858, 2010.

Yamamori, M. and Sato, K.: Characteristics of inertia gravity waves over the South Pacific as revealed by radiosonde obeservations, J. Geophys. Res., 111, D16110, doi:10.1029/2005JD006861, 2006.

Yoshiki, M. and Sato, K.: A statistical study of gravity waves in the polar regions based on operational radiosonde data, J. Geophys. Res., 105, 17995-18011, 2000. 
Zhang, F., Wang, S., and Plougonven, R.: Uncertainties in using the hodograph method to retrieve gravity wave characteristics from individual soundings, Geophys. Res. Lett., 31, L11110, doi:10.1029/2004GL019841, 2004.
Zhou, X., Holton, J. R., and Mullendore, G. L.: Forcing of secondary waves by breaking of gravity waves in the mesosphere, J. Geophys. Res., 107, 4058, doi:10.1029/2001JD001204, 2002. 